\title{
Rheology of granular materials composed of crushable particles
}

\author{
Duc-Hanh Nguyen ${ }^{1,2,3, a}$, Émilien Azéma ${ }^{1}$, Philippe Sornay ${ }^{2}$, and Farhang Radjaï ${ }^{1,4}$ \\ 1 LMGC, Univ. Montpellier, CNRS, Montpellier, France \\ 2 CEA, DEN, DEC, SFER, LCU, F-13108 Saint-Paul-les-Durance, France \\ 3 Faculty of Hydraulic Engineering, National University of Civil Engineering, Hanoi, Vietnam \\ $4\langle\mathrm{MSE}\rangle^{2}$, UMI 3466 CNRS-MIT, MIT Energy Initiative, 77 Massachusetts Avenue, 02139 Cambridge, MA, USA
}

\begin{abstract}
We investigate sheared granular materials composed of crushable particles by means of contact dynamics simulations and the bonded-cell model for particle breakage. Each particle is paved by irregular cells interacting via cohesive forces. In each simulation, the ratio of the internal cohesion of particles to the confining pressure, the relative cohesion, is kept constant and the packing is subjected to biaxial shearing. The particles can break into two or more fragments when the internal cohesive forces are overcome by the action of compressive force chains between particles. The particle size distribution evolves during shear as the particles continue to break. We find that the breakage process is highly inhomogeneous both in the fragment sizes and their locations inside the packing. In particular, a number of large particles never break whereas a large number of particles are fully shattered. As a result, the packing keeps the memory of its initial particle size distribution, whereas a power-law distribution is observed for particles of intermediate size due to consecutive fragmentation events whereby the memory of the initial state is lost. Due to growing polydispersity, dense shear bands are formed inside the packings and the usual dilatant behavior is reduced or cancelled. Hence, the stress-strain curve no longer passes through a peak stress, and a progressive monotonic evolution towards a pseudo-steady state is observed instead. We find that the crushing rate is controlled by the confining pressure. We also show that the shear strength of the packing is well expressed in terms of contact anisotropies and force anisotropies. The force anisotropy increases while the contact orientation anisotropy declines for increasing internal cohesion of the particles. These two effects compensate each other so that the shear strength is nearly independent of the internal cohesion of particles.
\end{abstract}

\section{Introduction}

Particle damage and fracture occur in granular materials both in industrial applications such as powder technology and mining and in natural rock and soil deformations and flows. Particle size reduction during such processes affects the mechanical behavior of a granular material through both energy dissipation and evolution of granular texture [1-4]. The manufacture of compact shapes by molding powders is a well-known example in which the bulk crushing of particles plays as much a role as particle rearrangements [5-9]. The grinding process of clinker nodules in the cement industry is another example where particle crushing is used to produce the desired size grading. The energetic (in)efficiency of such operations is a crucial issue that needs to be improved by a better control of the fragmentation process [9]. Another important example is the effect of particle crushing on stress distribution in earth dams. The permeability of soils constituting dams is reduced by particle breakage, thus increasing pore pressure and dam failure probability by soil liquefaction [10].

Despite its industrial importance, the particle breakage process and its underlying microscopic mechanisms are still poorly understood due to granular disorder and short length and time scales governing particle breakage. For example, it is experimentally observed that crushing may begin at stresses much lower than the cohesive strength of single particles $[3,11]$. This is a purely granular effect that reflects stress concentration due to the presence of strong force chains. At low confining stresses, tensile fracturing plays an important role in the development of shear bands $[12,13]$. In such cases, particle fracture occurs mainly in intense shearing bands, and the mechanical properties of the granular sample become strongly inhomogeneous. Conversely, the advent of particle breakage may be at the origin of shear banding.

A key issue is whether a self-similar or stable size distribution is reached under continuous fragmentation by 
shearing or compaction and whether the memory of the initial size distribution is lost by the fragmentation process [14]. Several experiments seem to indicate that particle fragmentation in granular materials does not significantly change the size of the largest particles (though their number declines) [14-17]. In fact, the larger particles are mostly surrounded by smaller particles, reducing thus the mean shear stress that they support. In this way, the lower shear stress exerted on large particles outweighs the decreasing strength of individual particles with increasing particle size. For this reason, the resulting size distribution is expected to be dependent on the initial size distribution.

Analytical models have also been proposed for the evolution of the particle size distribution (PSD) [18-24]. Most models are based on kinematic considerations and the cascading nature of the fragmentation process, leading to lognormal distributions or power laws [20, 25, 26] A powerlaw distribution of fragment sizes may be a consequence of the absence of characteristic length scales in the process $[27,28]$. This power-law PSD, as observed in experiments, is sometimes attributed to the force network [29]. However, the force network has not a fractal nature, although the inhomogeneous distribution of forces, composed of a network of strong force chains embedded in a network of weak forces carrying low shear stress [8,30-36], plays a prominent role in the process of particle crushing $[19,24]$.

Numerical simulations by Discrete-Element Methods (DEM) have been increasingly employed in order to get a better understanding of the particle size reduction [37-41]. Such discrete element numerical methods, based on rigidbody dynamics and frictional contact interactions, require a model of particle fracture such as the Bonded Parti-cle Model (BPM) in which the particles are modeled as aggregates of spherical sub-particles bonded by cohesive forces $[3,4,17,42-50]$. In this model, the contacts between sub-particles need to be calibrated to produce a targeted macroscopic behavior of the material $[42,43]$. DEM simulations of crushable granular materials have provided many interesting insights about the mechanisms of fracture propagation and effects of particle crushing. It is remarkable that, even in simple BPM simulations, a rather good agreement is often observed with experimental data. For example, the size-dependent strength of single particles is correctly reproduced $[9,45]$.

More recently, new variants of DEM-based fracture methods have been developed by accounting more rigorously for the fracture mechanics of individual particles. The particles are divided into cells by Finite-Element (FE) meshing $[13,51,52]$ and the potential fracture paths are simply represented by damageable cohesive interface elements. Alternatively, the cells can be treated as rigid elements of polygonal or polyhedral shapes obtained by Voronoi tessellation of the particles [53-57]. In this Bonded-Cell Model (BCM), the cells pave the whole volume of the particle so that the total volume is conserved by particle crushing. Recently, it was shown that this model may be simulated by means of the Contact Dynamics (CD) method by assuming that the cells are perfectly rigid and their common sides are governed by frictional- cohesive contacts [57]. The CD method accounts for the correct kinematics of side-side contacts. A crack is generated only when all critical intercellular contacts (contacts at their tensile threshold) percolate across the particle. This model reproduces correctly major fracture properties of disk-like particles subjected to diametrical compression, including fracture modes, size dependence and compressive strength [57].

In this paper, we use CD simulations with the BCM to analyze the behavior of granular samples composed of many crushable pentagonal particles subjected to biaxial shearing. We are interested in the stress-strain behavior and the evolution of PSDs. In particular, in contrast to shear without taking into account particle breakage, the value of the confining stress plays an important role and it will be quantified in this study.

During biaxial compression, the packing deforms due to both particle rearrangements and particle crushing depending on the internal cohesion of the particles compared to the confining pressure. The continuous shearing of a granular assembly under biaxial compression implies also the redistribution of the fragments generated by particle crushing and hence their efficient filling of the void space. It is therefore interesting to understand how this fragmentation process affects the evolution of the internal friction coefficient and dilatancy of the granular samples.

In the following, we first briefly describe the numerical approach and simulation parameters. Then, we focus on the stress-strain behavior. Finally, we analyze the evolution of the samples in terms of PSD and texture. We conclude with salient results of this work.

\section{Numerical approach}

\subsection{Bonded-cell model}

The simulations were performed by means of the CD method together with the BCM as detailed in [57]. We used particles of polygonal shape which were divided into $n_{v}$ cells by Voronoi tessellation, each cell representing a rigid sub-particle. For a particle of area $S$, the number of cells is given by $n_{v}=S / d_{0}^{2}$, where $d_{0}$ is the average cell size. $n_{v}$ points are distributed randomly inside each particle by imposing that the distance $L$ between the points is above $0.8 d_{0}$. The cells interact via cohesive frictional contacts along their common sides. A side-side contact between two cells involves two unilateral constraints, and hence two repulsive forces along the common side are required to prevent their overlap. For this reason, a side-side contact is represented by a set of two contact points, as illustrated in fig. 1(b), and the normal direction is the normal to the common side.

When a particle breaks into fragments, the latter may have both side-side and side-vertex contacts. The normal direction for a vertex-side contact is perpendicular to the cell side. Vertex-vertex contacts are rare, but when they occur, they are treated as double side-side contacts as detailed by Azema et al. [58]. In the CD method, the rigidbody equations of motion are integrated by simultaneously accounting for all kinematic constraints arising from 

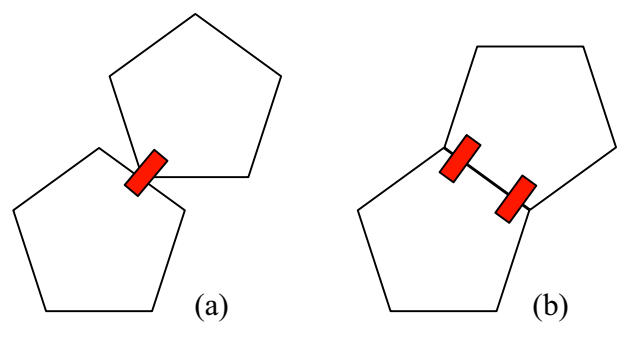

Fig. 1. Contact dynamics model of side-vertex (a) and sideside (b) contacts.

contact interactions [59-62]. The implicit time-stepping scheme allows for large time steps, and the numerical precision can be controlled independently by the number of iterations in each time step. The CD method has been extensively employed for the simulation of granular materials in $2 \mathrm{D}$ and $3 \mathrm{D}[30,59,63-78]$. But here it is applied for the first time to granular materials of crushable particles.

The cohesive strength $\sigma_{c}$ is assumed to be the same for all particles, and the shear strength is given by $\tau_{c}=\mu_{s} \sigma_{c}$, where $\mu_{s}$ is the internal friction coefficient. The choice of a frictional material behavior is not mandatory for the particles and $\tau_{c}$ may be defined independently of $\sigma_{c}$. We used a Coulomb friction law in order reduce the number of independent strength parameters. When the cohesion between two cells is lost along a side-side contact, the latter turns into a crack governed by the frictional contact behavior. The loss of cohesion is assumed to be irreversible.

\subsection{Simulation setup}

For our simulations we used pentagon-shaped particles. Due to their 5-fold directional symmetry, the pentagons are less prone to local ordering than hexagons and squares, which may spontaneously organize into locally ordered structures. The size of a pentagonal particle is defined by the diameter $d$ of its circumscribed circle. We used a uniform distribution of particle volume fractions in a range $\left[d_{\min }, d_{\max }=3 d_{\min }\right]$. Initially, we have 1000 particles, which may potentially fragment into 44249 fragments of diameter $d_{0}=0.2 d_{\min }$ using the BCM; see fig. 2 . These particles are initially placed on a square lattice in a rectangular box of dimensions $l_{0} \times h_{0}$ and deposited under the action of the gravity $g$. Then, the gravity is set to 0 and the packings are subjected to isotropic compression. The friction coefficient between particles and with the walls is set to zero during compression in order to obtain dense and isotropic packings as shown in fig. 2(a). The coefficient of friction between cells is set to $\mu_{c}=0.3$.

This isotropic samples are then subjected to vertical compression by downward displacement of the top wall at a constant velocity $\dot{y}$, where $y$ is the vertical length, for a constant confining stress $\sigma_{0}$ acting on the lateral walls. The friction coefficient $\mu$ is now set to 0.3 between particles and to zero with the walls. The zero friction with the walls prevents from stress gradients as those leading to the Janssen effect [79]. The vertical shear rate $\dot{\varepsilon}=\dot{y} / y$ is
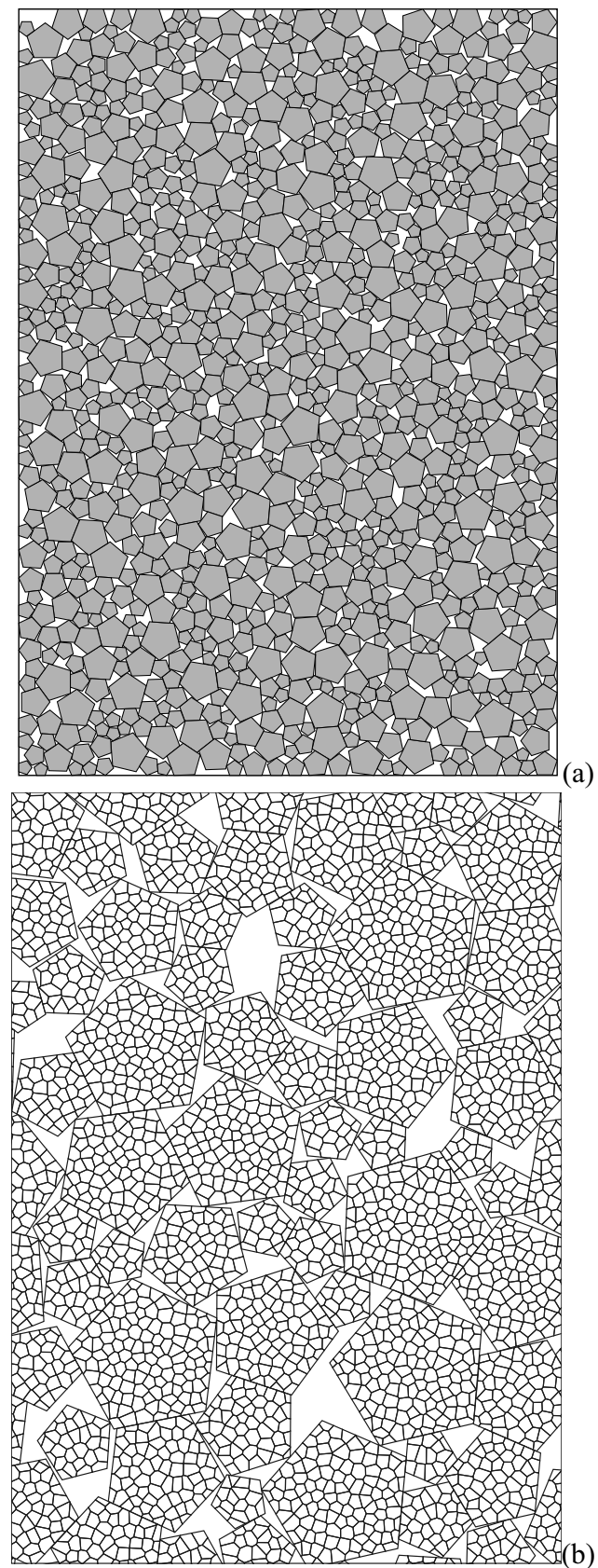

Fig. 2. Packing of pentagonal particles at the beginning of biaxial shearing (a); Particles tessellated into Voronoi cells (b).

low enough to ensure quasi-static conditions by reducing the value of the dimensionless inertia parameter $I$ given by (in 2D) [80] :

$$
I=\dot{\varepsilon} \sqrt{\frac{m}{p}}
$$

where $m$ is mean particle mass and $p$ is mean pressure. The quasi-static limit is characterized by the condition $I \ll 1$. In our simulations, $I$ was below $10^{-3}$.

For large values of the confining stress $\sigma_{0}$ compared to the internal cohesion $\sigma_{c}$, some particles break at the very beginning of the test. Since we are not interested here 
in particle crushing by the action of the applied stress ${ }^{1}$ we only consider cohesion levels such that the particles remain intact prior to the application of shear strain. This depends on the dimensionless number

$$
C_{e}=\frac{\sigma_{c}}{\sigma_{0}}
$$

which defines the "relative" cohesion of the particles. We checked that the samples are intact at the beginning of biaxial compression for $C_{e}>1$. Hence, we will analyze below the macroscopic behavior and particle fragmentation process for $C_{e}=1,2,5,10$ and 20, respectively. Since the confining stress is too low to cause spontaneous particle crushing, the latter occurs in response to shearing and fluctuating stresses. As we shall see below, the relative cohesion determines the crushing rate. Video samples of the simulations analyzed below can be found by following the link: www.cgp-gateway.org/ref 034 .

\section{Macroscopic behavior}

In this section, we consider the stress-strain and volumechange behavior for different values of the cohesion parameter $C_{e}$. We need to evaluate the stress tensor and packing fraction during deformation from the simulation data. For the stress tensor, we start with the tensorial moment $\boldsymbol{M}^{i}$ of each particle $i$ defined by $[81,82]$ :

$$
M_{\alpha \beta}^{i}=\sum_{c \in i} f_{\alpha}^{c} r_{\beta}^{c}
$$

where $f_{\alpha}^{c}$ is the $\alpha$ component of the force exerted on particle $i$ at contact $c, r_{\beta}^{c}$ is the $\beta$ component of the position vector of the same contact $c$, and the summation runs over all contact neighbors of particle $i$ (noted briefly by $c \in i$ ). The average stress tensor $\boldsymbol{\sigma}$ in the volume $V$ of the sample is given by the sum of tensorial moments of all particles divided by $V$ :

$$
\boldsymbol{\sigma}=\frac{1}{V} \sum_{i \in V} \boldsymbol{M}^{i}=\frac{1}{V} \sum_{c \in V} f_{\alpha}^{c} \ell_{\beta}^{c},
$$

where $\ell^{c}$ is the branch vector joining the centers of the two touching particles at contact point $c$. The first summation runs over all particles whereas the second summation runs over contacts.

Under biaxial compression, the major principal stress $\sigma_{1}$ is oriented along the compression axis whereas the minor principal stress $\sigma_{2}$ is perpendicular. The mean stress $p$ and stress deviator $q$ are defined as

$$
\begin{aligned}
& p=\frac{1}{2}\left(\sigma_{1}+\sigma_{2}\right), \\
& q=\frac{1}{2}\left(\sigma_{1}-\sigma_{2}\right) .
\end{aligned}
$$

\footnotetext{
1 The uniaxial compaction has been investigated using the same method but the results will be presented in an upcoming paper.
}
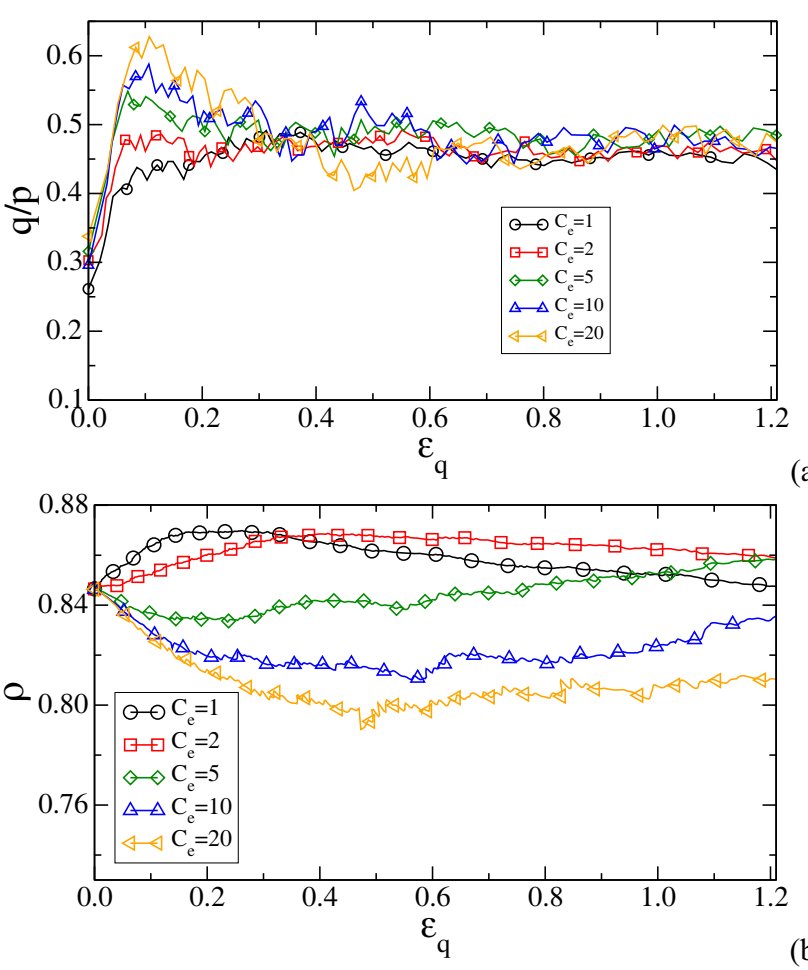

(a)

Fig. 3. Evolution of the normalized deviator stress $q / p$ (a) and packing fraction (b) as a function of the shear strain $\varepsilon_{q}$ for the different values of effective cohesion $C_{e}$.

For our system of perfectly rigid particles, the stress state is characterized by the mean stress $p$ and the normalized stress deviator $q / p$. The internal angle of friction is given by $\sin \varphi=q / p$.

The strain parameters are the cumulative vertical, horizontal and shear strains $\varepsilon_{1}, \varepsilon_{2}$ and $\varepsilon_{q}$, respectively. By definition, we have

$$
\varepsilon_{1}=\int_{h_{0}}^{h} \frac{\mathrm{d} h^{\prime}}{h^{\prime}}=\ln \left(1+\frac{\Delta h}{h_{0}}\right),
$$

where $h_{0}$ is the initial height and $\Delta h=h_{0}-h$ is the total downward displacement, and

$$
\varepsilon_{2}=\int_{l_{0}}^{l} \frac{\mathrm{d} l^{\prime}}{l^{\prime}}=\ln \left(1+\frac{\Delta l}{l_{0}}\right)
$$

where $l_{0}$ is the initial box width and $\Delta l=l-l_{0}$ is the total change of the box width. The cumulative shear strain is then defined by

$$
\varepsilon_{q} \equiv \varepsilon_{1}-\varepsilon_{2} .
$$

Finally, the cumulative volumetric strain $\varepsilon_{p}$ is given by

$$
\varepsilon_{p}=\varepsilon_{1}+\varepsilon_{2}=\int_{V_{0}}^{V} \frac{\mathrm{d} V^{\prime}}{V^{\prime}}=\ln \left(1+\frac{\Delta \rho}{\rho}\right)
$$

where $V_{0}=l_{0} h_{0}$ is the initial volume and $\Delta \rho=\rho-\rho_{0}$ is the cumulative change of packing fraction $\rho$.

Figure 3(a) shows the evolution of the normalized stress deviator $q / p$ as a function of the shear strain $\varepsilon_{q}$ 

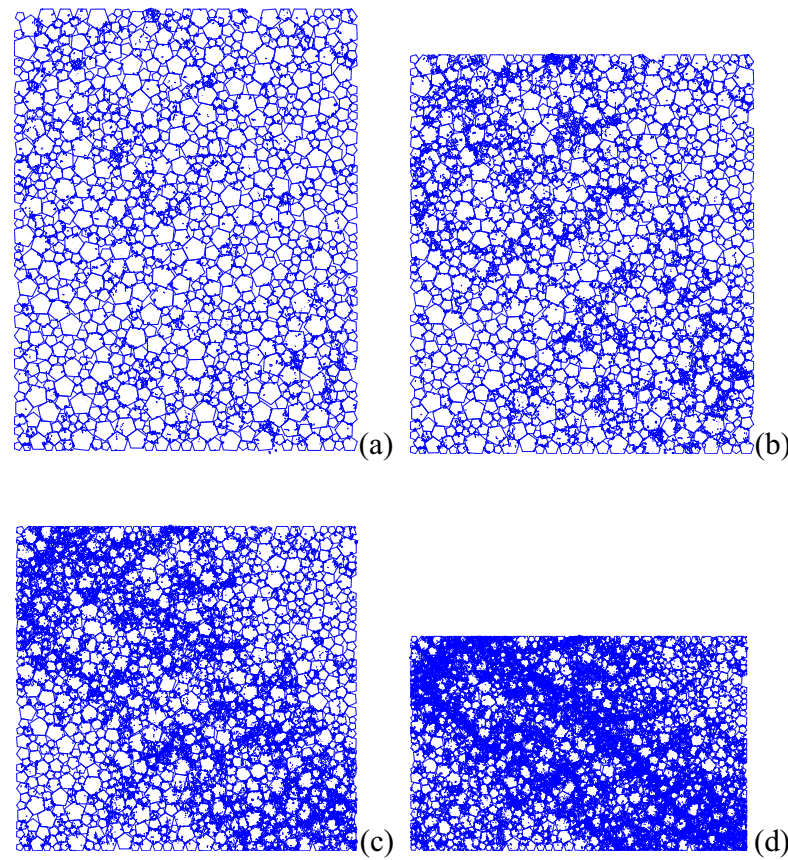

Fig. 4. Snapshots of the packing for $C_{e}=5$ during shear: (a) $\varepsilon_{q}=0.1$, (b) $\varepsilon_{q}=0.2$, (c) $\varepsilon_{q}=0.4$, (d) $\varepsilon_{q}=0.8$.

for different values of $C_{e}$. We see that for high values of $C_{e}, q / p$ increases to a peak before relaxing to a constant value $q^{*} / p \equiv \sin \varphi^{*}$ in steady shearing state. For the lowest values of $C_{e}, q / p$ evolves to the same steady value $\sin \varphi^{*}$ without passing by a peak. The peak stress increases with $C_{e}$ as observed by triaxial shear tests [83]. Hence, a major effect of enhanced particle crushing due to low cohesion of particles, as we shall see below, is to cancel or reduce the stress peak as the packing is sheared.

Figure 3(b) shows the evolution of packing fraction as a function of $\varepsilon_{q}$. Here again, we clearly observe the effect of particle fragmentation, which leads to a contracting behavior (increase of $\rho$ ) at low cohesion and to a dilating behavior (decrease of $\rho$ ) at high cohesion at the beginning of shearing. Particle rearrangements induced by shear imply dilation since our samples are initially very dense, but this dilation is counter-balanced here by the fragmentation of particles when the cohesion is weak, tending thus to increase the packing fraction by numerous small particles filling the space between larger particles. Interestingly, the evolution of $\rho$ is subsequently reversed by crushing in the dilatant case and by dilation in the contracting case. All samples seem to converge towards the same level of packing fraction. Nevertheless, a constant packing fraction is not reached. For this reason, we will refer below to this state as "quasi-steady" state.

The long evolution of the packing fraction reflects the fact that the breaking process is not homogeneous. Particle breakage occurs initially in the entire volume, but it quickly concentrates in several shear bands as observed in a series of snapshots in fig. 4 for $C_{e}=5$. A zoom on a shear band is displayed in fig. 5. We see that the shear bands are mainly composed of fragments and damaged

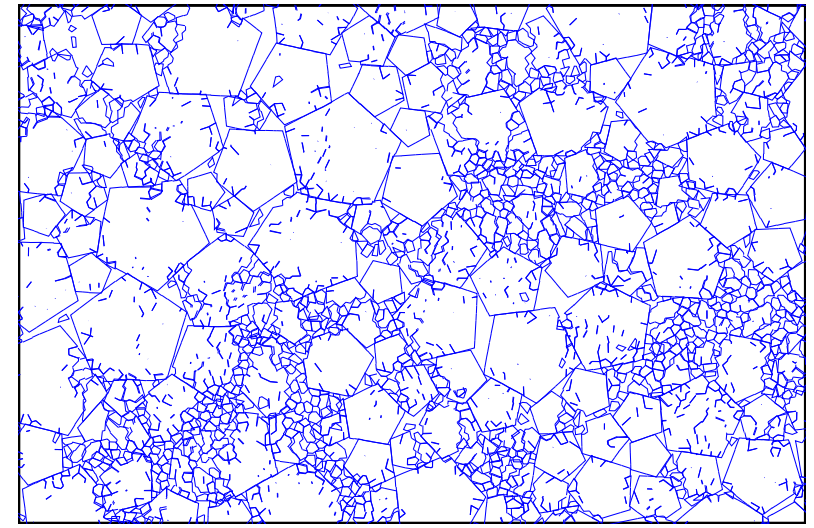

Fig. 5. Zoom on a shear band at $\varepsilon_{q}=0.4$. The line segments are cracks.

particles. A close inspection of the snapshots also reveals that the cracks often occur at the boundaries of particles, where the stresses are mostly concentrated by side-vertex contacts.

Another striking feature of the shear bands is the shattering of many particles whereas some others remain nearly intact. This indicates that particle crushing is often an unstable process in which strong force chains remain active on a damaged or fragmented particle until it fully breaks up. On the other hand, many small fragments produced from the breakup of particles in the neighbourhood of a large particle may reduce the mean shear stress acting on the large particle and hence its probability of failure. As a consequence of this coexistence between intact and shattered particles, the packing fraction is higher in the shear zones. This feature is also unique to a packing of crushable particles since the shear bands in the absence of particle breakage are often dilatant zones with a packing fraction below the average [84-88].

\section{Evolution of particle damage and size distributions}

In order to achieve a quantitative description of the fragmentation process, we need to analyze the proportions of cracks, damaged particles and fragment sizes during shear. Figure $6(\mathrm{a})$ shows the proportion $P_{b}$ of broken (or damaged) particles as a function of cumulative shear strain. This is the proportion of particles that have undergone any degree of damage (one or several cracks or broken into two or more fragments). $P_{b}$ increases rapidly at the beginning of shear and then continues to increase at a lower rate in the quasi-steady state for $\varepsilon_{q} \simeq 0.4$. It is remarkable that the plots nearly coincide for $C_{e} \leq 5$. For lower values of $C_{e}$ nearly all particles are damaged before a quasi-steady state is reached. This suggests that, even at these levels of cohesion for which the particles do not break at the beginning of shear under the action of the confining stress, the latter prevails nevertheless during shear. In contrast, for $C_{e} \geq 5$, the latter plays no role and particle breakage appears to be fully governed by shearing. 


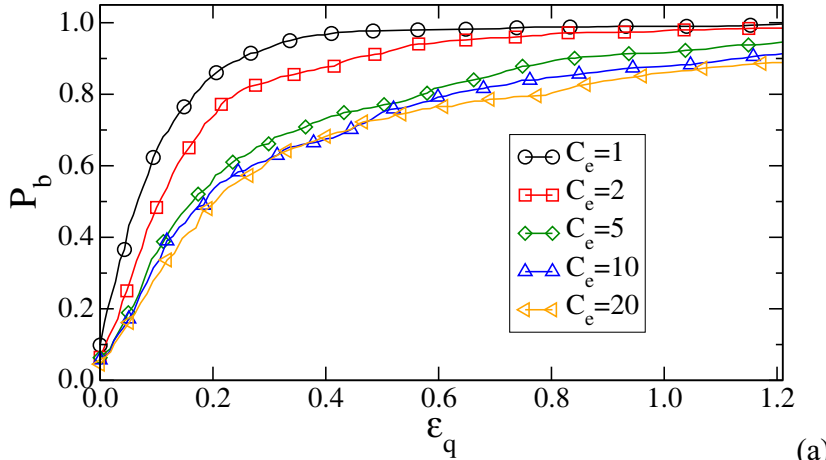

(a)

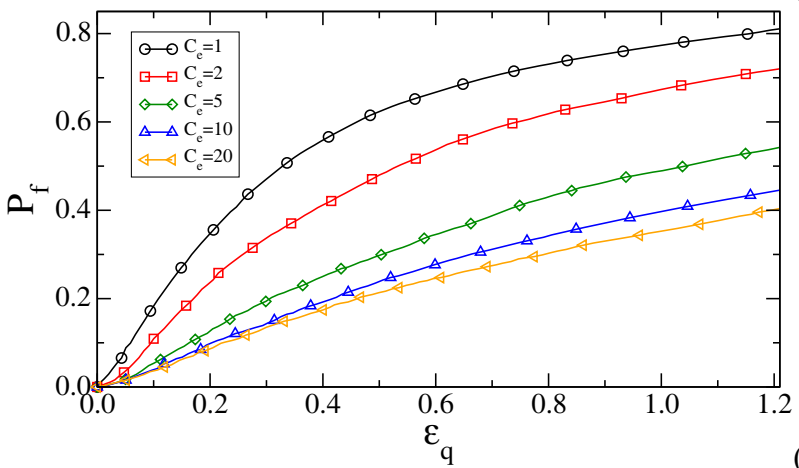

(b)

Fig. 6. Evolution of the proportion $P_{b}$ of damaged particles (a) and the fraction $P_{f}$ of broken contacts (b) as a function of cumulative shear strain during biaxial compression for different values of $C_{e}$.

It is also important to note that even for $C_{e} \leq 5$ all cohesive contacts do not break apart and all particles are not fully fragmented. Figure $6(\mathrm{~b})$ shows the proportion $P_{f}$ of broken contacts. $P_{f}$ increases steadily such that no difference can be observed between the initial and late regimes as we observed in the evolution of $P_{b}$. This means that particle damage is a more sensitive descriptor of the process than contact breakage. The relative cohesion $C_{e}$ controls the rate of contact breakage but not that of particle breakage, which seems to be independent of $C_{e}$ for $C_{e} \leq 5$ and is more closely correlated with the evolution of the stress deviator.

The cumulative volume fraction $(\mathrm{CVF}) h(d)$ of particle fragments (total volume of particles with diameters below $d$ ) is shown in fig. 7 for two values of $C_{e}$. The initial distribution is uniform by particle volume fractions, corresponding to an affine function in this representation of PSD. We observe a progressive evolution of $h$ towards smaller particle diameters. The distribution $h$ remains essentially uniform by particle volume fractions for all diameters except for fragments of diameters close to the size of cells. Since the cells cannot break, the number of cell-size fragments increases during this process.

We may thus characterize the size distributions as composed of three distinct parts: 1) affine with a high slope $\beta$ for $\left.d / d_{\max } \geq 0.3,2\right)$ affine with a lower slope for $0.1 \leq d / d_{\max } \leq 0.3$ and 3 ) a population of particles with a size of about the mean cell size for $d / d_{\max } \leq 0.1$. Obviously, the affine shape of the CVF implies that the frag-
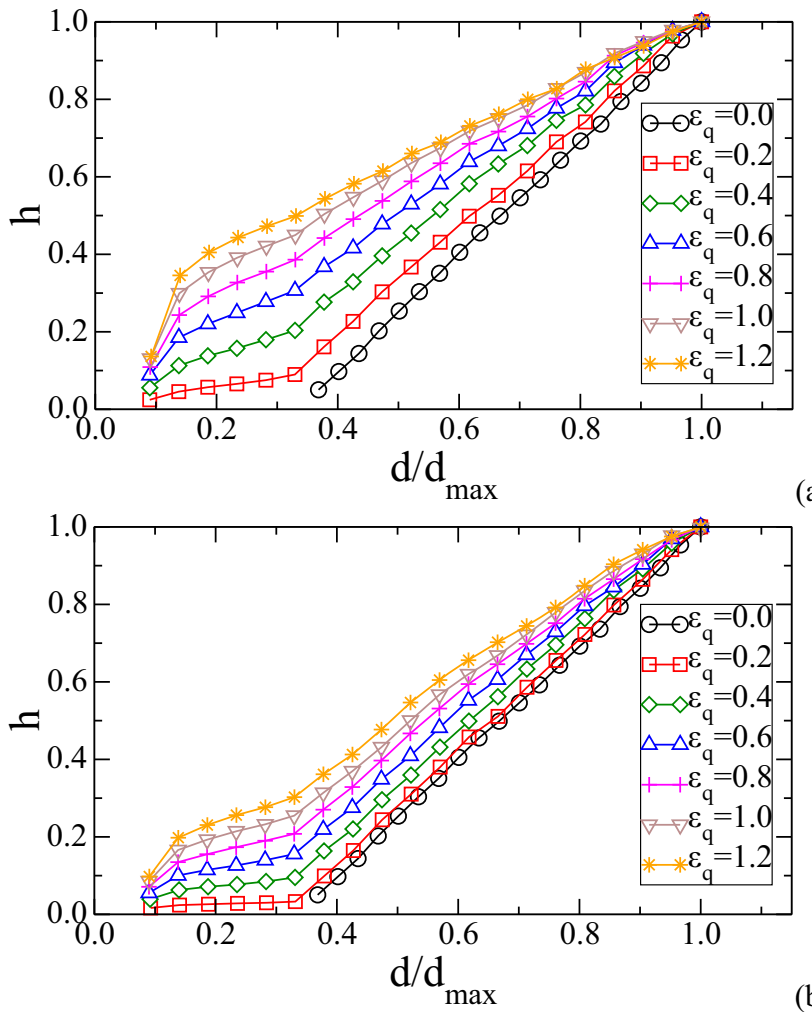

(a)

(b)

Fig. 7. Evolution of the cumulative volume fraction of particles during the biaxial deformation for $C_{e}=5$ (a) and $C_{e}=20$ (b)

ment sizes are distributed as $P(d) \propto d^{-2}$ initially and in the range of both large and small fragments. The exponent $D=-2$ is the fractal dimension. Our value of the fractal dimension is close to many others simulation and experimental results [89-92] with values ranging from 1.5 to 3.5 for granular materials. The first part is the reminiscence of the initial size distribution. In particular, the largest particle size $d_{\max }$ is not reduced even at large shear strains. This part of the distribution consists mainly of particles that have lost small fragments. The second affine distribution is the population of fragments generated from the breakup of particles. Its affine shape can be attributed to the self-similar nature of the process, which allows for fragments of all sizes to be generated from larger particles [89].

The first part of the CVF (large fragments) can thus be approximated as

$$
h(d)=h\left(d_{1}\right)+\beta\left(d-d_{1}\right)
$$

where $d_{1} \simeq 0.4 d_{\text {max }}$ is the particle size separating the two affine parts and

$$
\beta=\frac{1-h\left(d_{1}\right)}{d_{\max }-d_{1}}
$$

The volume fraction $h\left(d_{1}\right)$ increases with $\varepsilon_{q}$ and it declines when $C_{e}$ increases. Figure 8 shows the evolution of $\beta$ with $\varepsilon_{q}$ for different values of $C_{e}$. Just as the proportion of damaged particles, $\beta$ decreases first rapidly depending on the value of $C_{e}$ and then continues to decrease steadily at 


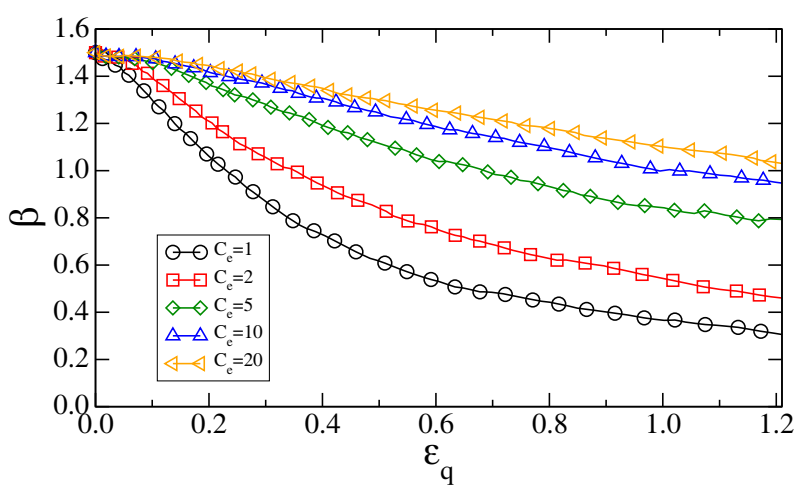

Fig. 8. Evolution of parameter $\beta$ (see text) as a function of cumulative shear strain for different values of effective cohesion.

a lower rate with nearly the same rate for all values of $C_{e}$. The size reduction rate in this phase reflects the shear rate. This is fully consistent with the picture that the evolution of the packing is governed by pressure-induced crushing at the beginning of biaxial shearing and by strain rate in the quasi-steady state.

\section{Contact network and fabric evolution}

It was shown above that the internal angle of friction in the quasi-steady state is nearly constant while particles keep breaking and the microstructure and packing fraction evolve. In this section, we analyze the microstructure and contact variables such as force and fabric anisotropies in order to gain a deeper insight about physical mechanisms underlying the shear strength in a granular packing of crushable particles. Figure 9 shows successive snapshots of a portion of the contact network during shear. The microstructure can be described in terms of the connectivity of the contact network, which at lowest order is characterized by the coordination number $Z$ (average number of contact neighbours per particle), and the orientations $\boldsymbol{n}$ of contacts. We see that the contact network changes as a result of the generation of new fragments of different sizes. These fragments move in the course of shearing. The query here is whether the granular texture is influenced only through the increasing polydispersity of the particles.

Figure 10 shows the evolution of $Z$ as a function of shear strain. As in the case of packing fraction analyzed in sect. 3 , for $C_{e}>5$ we find here an unmonotonic evolution due to competition between volume change (dilation or contraction) by particle rearrangements and enhanced space-filling by small fragments as a result of particle fragmentation. For $C_{e}<5, Z$ declines monotonically. This may appear surprising since the packing fraction increases at the beginning of shear. It indicates that all generated fragments do not spontaneously make new contacts with existing particles and their fragments. As for packing fraction, the contact network even after large shearing keeps changing despite the nearly constant value of the shear strength.

The anisotropies of the contact network and contact forces are usually described in terms of the probability density function $P(\boldsymbol{n})$ of contact normals, the average
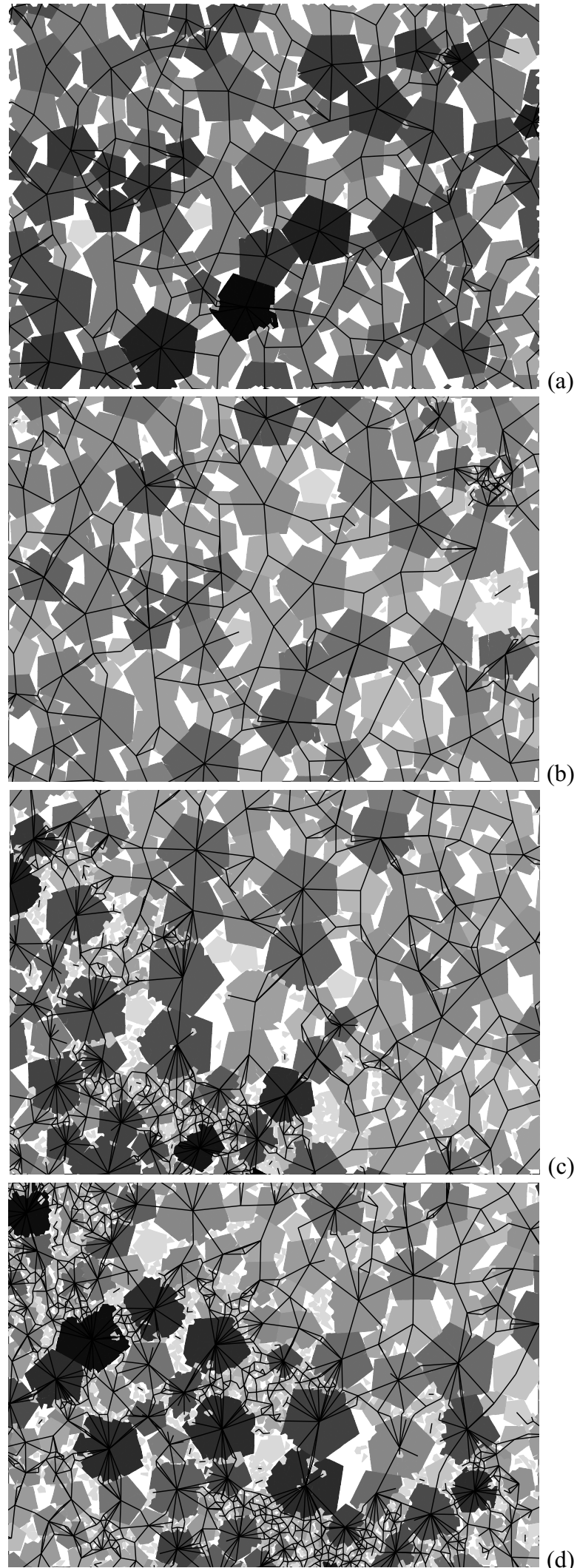

Fig. 9. Snapshots of a portion of the contact network for $C_{e}=20$ at $\varepsilon_{q}=0.1$ (a), $\varepsilon_{q}=0.2(\mathrm{~b}), \varepsilon_{q}=0.6$ (c) and $\varepsilon_{q}=1.0$ (d). The contacts are represented by segments joining particle centers. The gray level of particles is proportional to the coordination number. 


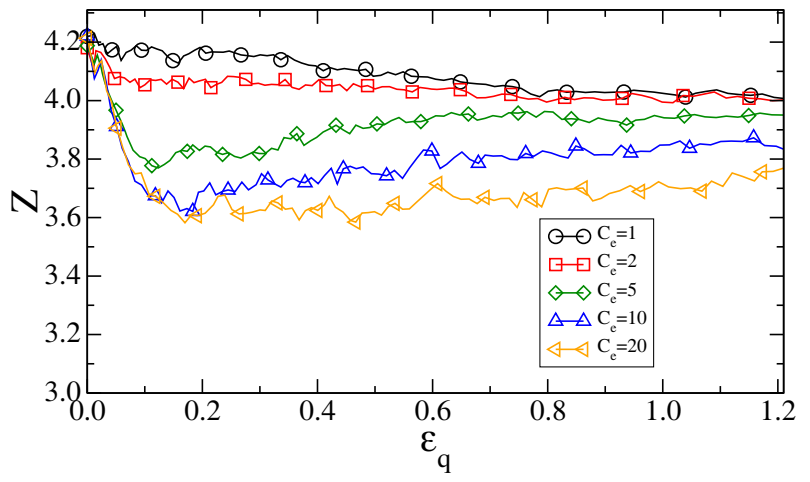

Fig. 10. Evolution of the coordination number $Z$ as a function of shear strain for different values of $C_{e}$.

branch vector (joining particle centers) $\langle\boldsymbol{\ell}\rangle(\boldsymbol{n})$ and the average contact force $\langle\boldsymbol{f}\rangle(\boldsymbol{n})$ as a function of the contact normal $\boldsymbol{n}$ [61,93-98]. In two dimensions, the unit vector $\boldsymbol{n}$ is defined by its angle $\theta$ with the $x$-axis, and the branch vector $\boldsymbol{\ell}$ and contact force $\boldsymbol{f}$ can be represented by their normal and tangential components. Thus, the probability density $P_{\theta}(\theta)$ of contact orientations $\theta$, the angular averages of the components $\left\langle\ell_{n}\right\rangle(\theta)$ and $\left\langle\ell_{t}\right\rangle(\theta)$ of the branch vector and the average components $\left\langle f_{n}\right\rangle(\theta)$ and $\left\langle f_{t}\right\rangle(\theta)$ of forces as a function of contact orientation $\theta$ provide a full description of the anisotropic state.

As a granular material is sheared, the above functions tend to take a simple unimodal shape well approximated by their truncated $\pi$-periodic Fourier expansions $[93,96]$ :

$$
\left\{\begin{array}{l}
P(\theta)=\frac{1}{2 \pi}\left\{1+a_{c} \cos 2\left(\theta-\theta_{c}\right)\right\} \\
\left\langle\ell_{n}\right\rangle(\theta)=\left\langle\ell_{n}\right\rangle\left\{1+a_{l n} \cos 2\left(\theta-\theta_{l n}\right)\right\} \\
\left\langle\ell_{t}\right\rangle(\theta)=\left\langle\ell_{n}\right\rangle a_{l t} \sin 2\left(\theta-\theta_{l t}\right), \\
\left\langle f_{n}\right\rangle(\theta)=\left\langle f_{n}\right\rangle\left\{1+a_{f n} \cos 2\left(\theta-\theta_{f n}\right)\right\} \\
\left\langle f_{t}\right\rangle(\theta)=\left\langle f_{n}\right\rangle a_{f t} \sin 2\left(\theta-\theta_{f t}\right),
\end{array}\right.
$$

where $a_{c}$ is the contact orientation anisotropy, $a_{l n}$ is the normal branch anisotropy, $a_{l t}$ is the tangential branch anisotropy, $a_{f n}$ is the normal force anisotropy, and $a_{f t}$ is the tangential force anisotropy. The angles $\theta_{c}, \theta_{l n}, \theta_{l t}$, $\theta_{f n}$, and $\theta_{f t}$ are the corresponding privileged directions. Here, we consider all contacts between cells belonging either to two different particles or to the same particle. All cell-cell contacts transmit forces and, for this reason, they are involved in the average stress tensor of the packing.

Figure 11(a) shows the evolution of the contact orientation anisotropy $a_{c}$ for different values of $C_{e}$. For all values of $C_{e}, a_{c}$ increases with shear strain. For $C_{e}=1$ and $C_{e}=5$ we observe a fast increase of anisotropy at the beginning, followed by a slow increase above $\varepsilon_{q}=0.4$. Particle fragmentation affects therefore the contact network by an increase of $a_{c}$ (and decrease of $Z$ as it was shown before). Figures 11(b) and (c) show the anisotropies of normal forces $a_{f n}$ and tangential forces $a_{f t}$ as a function of shear strain. Both anisotropies increase to a peak, and then decrease and level off at a constant value in the quasisteady state. During shear, the anisotropies are higher at higher values of $C_{e}$. The normal force anisotropy reflects

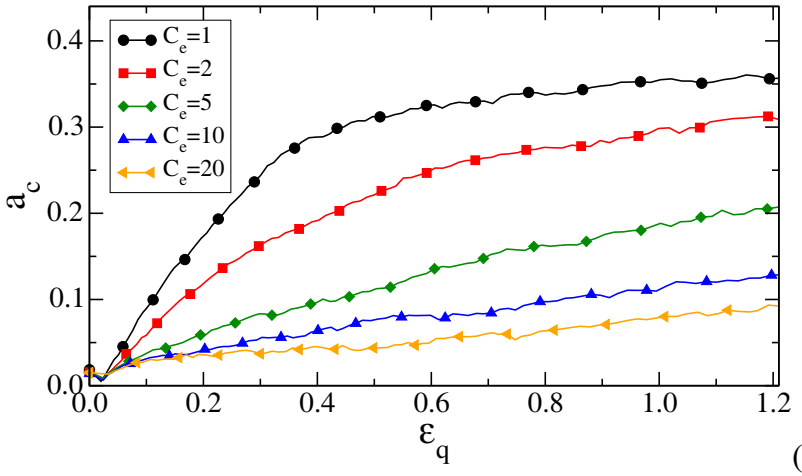

(a)

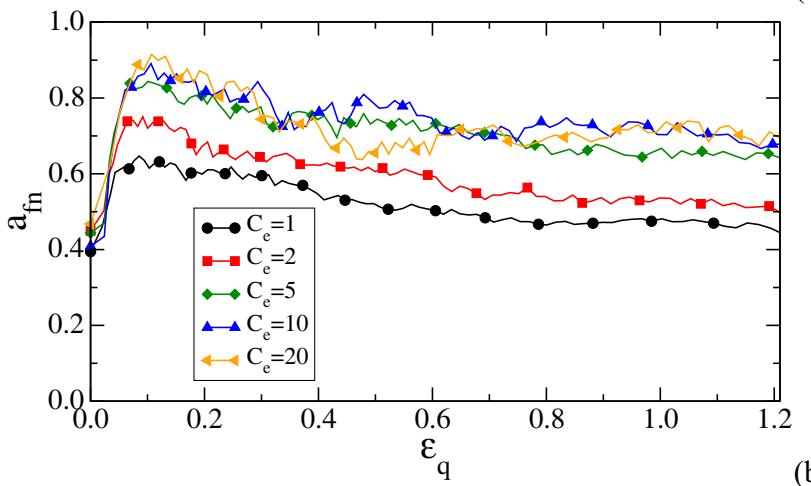

(b)

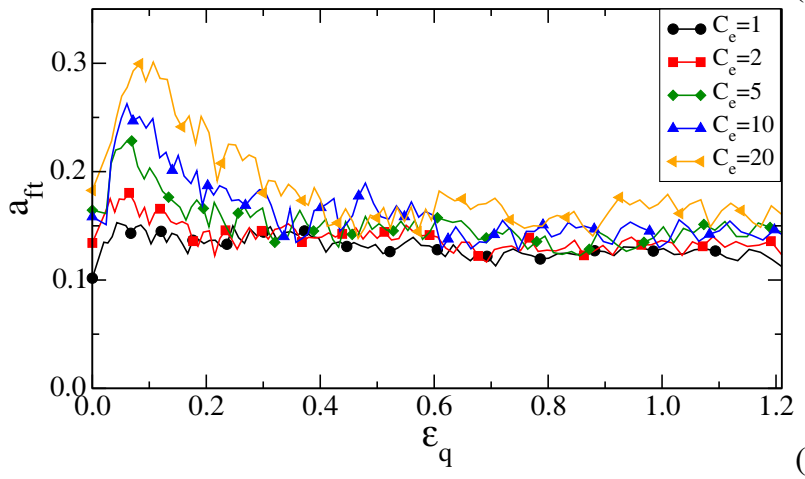

(c)

Fig. 11. Evolution of the contact orientation anisotropy $a_{c}$ (a), normal force anisotropy $a_{f n}$ (b) and tangential force anisotropy $a_{f t}(\mathrm{c})$ during shear.

strong force chains whereas the tangential force anisotropy is a measure of friction mobilization. In both cases, the anisotropy is lower at lower cohesion as particles break at too high force concentration or friction mobilization.

The internal angle of friction $\varphi^{*}$ in the quasisteady state is a direct consequence of fabric and force anisotropies. Due to shear, the privileged directions of contacts and forces tend to coincide with the major principal stress direction so that $\theta_{c} \simeq \theta_{l n} \simeq \theta_{l t} \simeq \theta_{f n} \simeq \theta_{f t}$. Under these conditions, it can be shown that $[70,93,99]$

$$
\sin \varphi^{*} \simeq \frac{1}{2}\left(a_{c}+a_{l n}+a_{l t}+a_{f n}+a_{f t}\right) .
$$

The evolution of $\sin \phi^{*}$ as well as its predicted value from the above relation are shown in fig. 12(a) as a function of $C_{e}$. We see that $\varphi^{*}$ is nearly independent of $C_{e}$ and the measured values are in excellent agreement with the predicted values. The evolution of different anisotropies with 


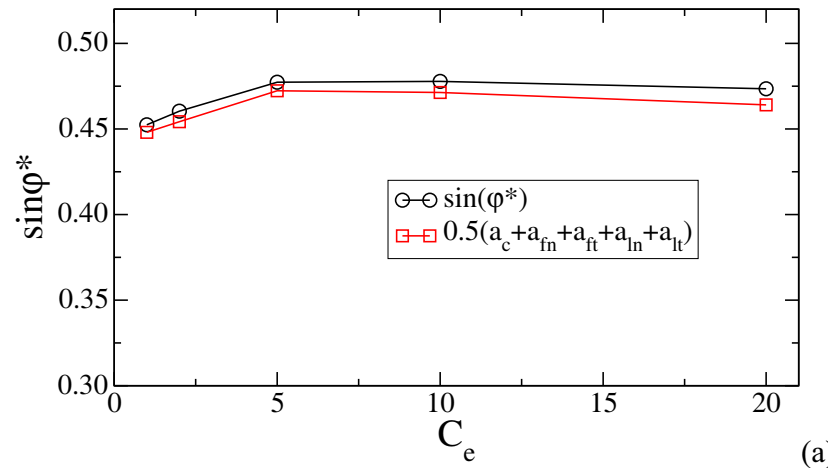

(a)

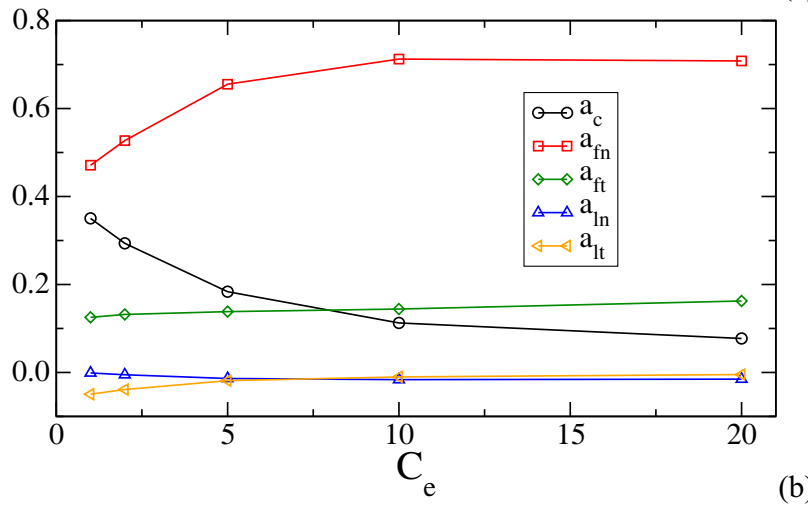

(b)

Fig. 12. Normalized shear stress $\sin \phi^{*}$ and harmonic approximations given by eq. (14) (a) and mean value of anisotropies in the quasi-steady state as a function of the effective cohesion $C_{e}$ (b).

$C_{e}$ is displayed in fig. 12(b). For increasing $C_{e}, a_{f n}$ increases and $a_{c}$ declines. The evolution of other anisotropies is not significant. Hence, the independence of $\varphi^{*}$ with respect to the internal cohesion of the particles is a consequence of additive compensation between the increase of $a_{f n}$ and the decrease of $a_{c}$. The increase of $a_{f n}$ may be attributed to the production of particles of anisotropic shape by fragmentation whereas the decrease of $a_{c}$ is a consequence of increasing polydispersity. This behavior was previously analyzed for polydisperse granular materials [74]. The shear strength is basically controlled by the largest particles, which capture strong force chains and lead to a higher force anisotropy whereas the contact anisotropy declines due to increasing polydispersity. Hence, the nature of force transmission and fabric anisotropy both change with the internal cohesion of particles on which the fragmentation process depends, but the shear strength remains nearly unchanged.

Note that the shear strength at peak $\sin \phi^{\text {peak }}$ increases strongly with $C_{e}$ as shown in fig. 13(a). We also see in fig. $13(\mathrm{~b})$ that $\sin \phi^{\text {peak }}$ increases mainly due to the increase of the normal force anisotropy $a_{f n}$ and the tangential force anisotropy $a_{f t}$ with $C_{e}$.

\section{Conclusion}

We used the bonded-cell model in the contact dynamics framework to investigate the mechanical behavior of pack-
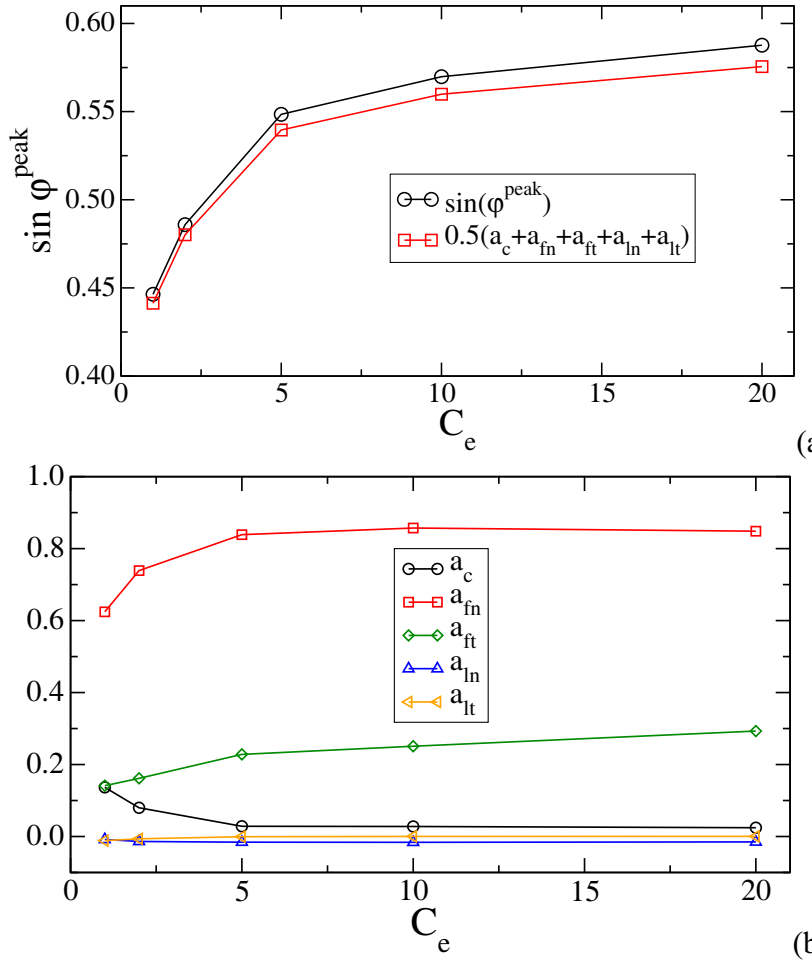

(a)

Fig. 13. Normalized shear stress $\sin \phi^{*}$ and harmonic approximations given by eq. (14) (a) and mean values of anisotropies at peak as a function of the effective cohesion $C_{e}(\mathrm{~b})$.

ings of pentagonal crushable particles subjected to biaxial shearing. In this work, the ratio of the mean confining stress to the internal cohesion of particles was fixed and the fragmentation process was a consequence of continuous shearing and local stress fluctuations. This is different from most reported studies in which particle crushing is a direct consequence of increasing stress. In this respect, in our simulations particle crushing is an intrinsic mechanism of shear together with particle rearrangements, sliding and rolling. Hence, the effect of particle crushing on the rheological behavior (shear strength, dilatancy) is a fundamental issue that was addressed in this paper. It is important to note that our breaking method by representing the particles as clusters of bonded cells, allows for particle breakage without loss of volume, whereas most investigations in the past used the bonded-particle method in which the particles are aggregates of spherical or circular particles.

It was shown that particle crushing can suppress dilatancy at low ratios of the internal cohesion of the particles to the confining stress. This ratio, which represents the relative cohesion of the particles, affects also the particle fragmentation rate. In all cases, a quasi-steady state is reached after a transient with a nearly constant shear strength although particles keep breaking by shear. We also analysed the force and fabric anisotropies underlying shear strength. The force anisotropy increases while the contact orientation anisotropy decreases with the internal cohesion of the particles. The mutual compensation of these anisotropies for increasing confining stress leads 
to a shear strength independent of confining stress in the quasi-steady state.

As to the evolution of particle size distribution, we found two complementary behaviors: 1) The memory of the particle size range is partially conserved since a fraction of large particles do not break, and 2) a power-law size distribution emerges in the range of intermediate fragment sizes due to loss of memory induced by the consecutive breakage of large particles into increasingly smaller fragments. For the lowest values of relative cohesion, the distribution is largely dominated by the effect of the irreducible size of the smallest fragments. For this reason, the size distributions of fragments for the lowest levels of cohesion are less reliable compared to real materials, wherein the fine particles may in principle continue to fragment and the size span can evolve. Another important feature evidenced by our simulations is the localization of strains and fragmentations in shear bands. Size polydispersity being greater in these bands, contrary to the dilatant bands usually observed, these bands are more compact and therefore resemble compaction bands. The width of these bands increases with the shear.

This work can be pursued for a better understanding of the effects of different geometrical parameters such as particle shapes and their initial size distributions. The initial particle shapes can be irregular polygons of arbitrary numbers of sides and aspect ratios. In particular, it would be both interesting and of practical interest to clarify the respective roles of the number of sides and aspect ratios, which affect both the initial value of the packing fraction and its anisotropic structure. The bonded-cell model can also be applied to 3D particles. We presently develop a 3D version of our approach, which, in application to representative packings of $3 \mathrm{D}$ particles, will require much higher computational effort than in $2 \mathrm{D}$.

We acknowledge financial support from the CEA.

\section{Author contribution statement}

The simulations reported and anlyzed in this paper were performed by Duc-Hanh Nguyen. All authors equally contributed to the analysis of the simulation results and writing of the present paper.

\section{References}

1. L. Elghezal, M. Jamei, I.-O. Georgopoulos, Granular Matter 15, 685 (2013).

2. Yukio Nakata, Masayuki Hyodo, Adrian F.L. Hyde, Yoshinori Kato, Hidekazu Murata, Soils Found. 41, 69 (2001).

3. Y.P. Cheng, Y. Nakata, M.D. Bolton, Géotechnique 53, $633(2003)$.

4. Y.P. Cheng, M.D. Bolton, Y. Nakata, Géotechnique 54, 131 (2004).

5. D.W. Fuerstenau, O. Gutsche, P.C. Kapur, Confined particle bed comminution under compressive loads, in Comminution 1994, edited by K.S.E. Forssberg, K. Schnert (Elsevier, Amsterdam, 1996) pp. 521-537.
6. C. Hosten, H. Cimilli, Int. J. Min. Process. 91, 81 (2009).

7. Arghya Das, Giang D. Nguyen, Itai Einav, J. Geophys. Res.: Solid Earth 116, B08203 (2011).

8. O. Ben-Nun, I. Einav, A. Tordesillas, Phys. Rev. Lett. 104, 108001 (2010).

9. V.P.B. Esnault, J.-N. Roux, Mech. Mater. 66, 88 (2013)

10. Poul V. Lade, Jerry A. Yamamuro, Paul A. Bopp, J. Geotech. Eng. 122, 309 (1996).

11. Fawad A. Chuhan, Arild Kjeldstad, Knut Bjørlykke, Kaare Høeg, Mar. Pet. Geol. 19, 39 (2002).

12. N. Cho, C.D. Martin, D.C. Sego, Int. J. Rock Mech. Min. Sci. 45, 1335 (2008)

13. Gang Ma, Wei Zhou, Xiao-Lin Chang, Comput. Geotech. 61, 132 (2014).

14. M.R. Coop, K.K. Sorensen, T. Bodas Freitas, G. Georgoutsos, Géotechnique 54, 157 (2004).

15. Charles Sammis, Geoffrey King, Ronald Biegel, Pure Appl. Geophys. 125, 777 (1987).

16. Luis E. Vallejo, Sebastian Lobo-Guerrero, Kevin Hammer, Int. J. Geomech. 6, 435 (2006).

17. Junyu Huang, Songlin Xu, Shisheng Hu, Mech. Mater. 68, 15 (2014).

18. K.H. Wohletz, M.F. Sheridan, W.K. Brown, J. Geophys. Res.: Solid Earth 94, 15703 (1989).

19. Sidney Redner, Statistical theory of fragmentation, in Disorder and Fracture (Springer, 1990) pp. 31-48.

20. J.A. Astrom, H.J. Herrmann, Eur. Phys. J. B 5, 551 (1998).

21. M. Gorokhovski, Fragmentation under the scaling symmetry and turbulent cascade with intermittency, Technical Report, DTIC Document, 2003.

22. Itai Einav, J. Mech. Phys. Solids 55, 1274 (2007).

23. Predrag Elek, Slobodan Jaramaz, FME Trans. 37, 129 (2009).

24. N.R.A. Bird, C.W. Watts, A.M. Tarquis, A.P. Whitmore, Vadose Zone J. 8, 197 (2009).

25. Ferenc Kun, Imre Varga, Sabine Lennartz-Sassinek, Ian G. Main, Phys. Rev. E 88, 062207 (2013).

26. Ferenc Kun, Imre Varga, Sabine Lennartz-Sassinek, Ian G. Main, Phys. Rev. Lett. 112, 065501 (2014).

27. Francesca Casini, Giulia M.B. Viggiani, Sarah M. Springman, Granular Matter 15, 661 (2013).

28. Benjy Marks, Itai Einav, Geophys. Res. Lett. 42, 274 (2015).

29. Luis E. Vallejo, Sebastian Lobo-Guerrero, Zamri Chik, $A$ network of fractal force chains and their effect in granular materials under compression, in Fractals in Engineering (Springer, 2005) pp. 67-80.

30. F. Radjaï, M. Jean, J.-J. Moreau, S. Roux, Phys. Rev. Lett. 77, 274 (1996).

31. Olivier Tsoungui, Denis Vallet, Jean-Claude Charmet, Stphane Roux, C. R. Acad. Sci., Ser. IIB 325, 457 (1997).

32. F. Radjaï, D.E. Wolf, M. Jean, J.J. Moreau, Phys. Rev. Lett. 80, 61 (1998).

33. C. Thornton, M.T. Ciomocos, M.J. Adams, Powder Technol. 140, 258 (2004)

34. Ivana Agnolin, Jean-Noël Roux, Phys. Rev. E 76, 061302 (2007).

35. Vincent Richefeu, Moulay Saïd El Youssoufi, Farhang Radjai, Phys. Rev. E 73, 051304 (2006).

36. C. Voivret, F. Radjaï, J.-Y. Delenne, M.S. El Youssoufi, Phys. Rev. Lett. 102, 178001 (2009). 
37. C. Thornton, K.K. Yin, M.J. Adams, J. Phys. D: Appl. Phys. 29, 424 (1996).

38. R. Moreno, M. Ghadiri, S.J. Antony, Powder Technol. 130, $132(2003)$.

39. L. Liu, K.D. Kafui, C. Thornton, Powder Technol. 199, 189 (2010).

40. Wei Zhou, Lifu Yang, Gang Ma, Kun Xu, Zhiqiang Lai, Xiaolin Chang, Granular Matter 19, 25 (2017).

41. Ming $\mathrm{Xu}$, Juntian Hong, Erxiang Song, Comput. Geotech. 89, 113 (2017) (Supplement C).

42. D.O. Potyondy, P.A. Cundall, Int. J. Rock Mech. Min. Sci. 41, 1329 (2004).

43. N. Cho, C.D. Martin, D.C. Sego, Int. J. Rock Mech. Min. Sci. 44, 997 (2007).

44. Manoj Khanal, Wolfgang Schubert, Jurgen Tomas, Miner. Eng. 20, 179 (2007).

45. M.D. Bolton, Y. Nakata, Y.P. Cheng, Géotechnique 58, 471 (2008).

46. Steffen Abe, Karen Mair, Geophys. Res. Lett. 36, L23302 (2009).

47. Jianfeng Wang, Haibin Yan, Soils Found. 52, 644 (2012).

48. G. Timár, F. Kun, H.A. Carmona, H.J. Herrmann, Phys. Rev. E 86, 016113 (2012)

49. Matthew J. Metzger, Benjamin J. Glasser, Powder Technol. 217, 304 (2012).

50. Takao Ueda, Takashi Matsushima, Yasuo Yamada, Granular Matter 15, 675 (2013).

51. S.A. Galindo-Torres, D.M. Pedroso, D.J. Williams, L. Li, Comput. Phys. Commun. 183, 266 (2012).

52. Gang Ma, Wei Zhou, Richard A. Regueiro, Qiao Wang, Xiaolin Chang, Powder Technol. 308, 388 (2017).

53. Ferenc Kun, Hans J. Herrmann, Comput. Methods Appl. Mech. Eng. 138, 3 (1996).

54. Bart Van de Steen, André Vervoort, J.A.L. Napier, Int. J. Fract. 108, 165 (2001).

55. G.A. D'Addetta, F. Kun, E. Ramm, Granular Matter 4 77 (2002).

56. S.A. Galindo-Torres, D.M. Pedroso, D.J. Williams, L. Li, Comput. Phys. Commun. 183, 266 (2012).

57. Duc-Hanh Nguyen, Emilien Azéma, Philippe Sornay, Farhang Radjai, Phys. Rev. E 91, 022203 (2015).

58. E. Azema, N. Estrada, F. Radjai, Phys. Rev. E 86, 041301 (2012).

59. J.J. Moreau, Eur. J. Mech. A Solids 13, 93 (1994).

60. M. Jean, Comput. Methods Appl. Mech. Eng. 177, 235 (1999).

61. Farhang Radjai, Vincent Richefeu, Mech. Mater. 41, 715 (2009).

62. Farhang Radjaï, Frédéric Dubois, Discrete Numerical Modeling of Granular Materials (Wiley-ISTE, New-York, 2011) ISBN: 978-1-84821-260-2.

63. L. Staron, J.-P. Vilotte, F. Radjaï, Phys. Rev. Lett. 89 , $204302(2002)$.

64. A. Taboada, K.J. Chang, F. Radjaï, F. Bouchette, J. Geophys. Res. 110, B09202 (2005).

65. M. Renouf, P. Alart, Comput. Methods Appl. Mech. Eng. 194, 2019 (2005).

66. E. Azéma, F. Radjaï, R. Peyroux, F. Dubois, G. Saussine, Phys. Rev. E 74, 031302 (2006).

67. E. Azéma, F. Radjaï, R. Peyroux, V. Richefeu, G. Saussine, Eur. Phys. J. E 26, 327 (2008)
68. Nicolas Estrada, Alfredo Taboada, Farhang Radjaï, Phys. Rev. E 78, 021301 (2008).

69. E. Azéma, F. Radjaï, Phys. Rev. E 81, 051304 (2010).

70. E. Azéma, F. Radjaï, Phys. Rev. E 85, 031303 (2012).

71. N. Estrada, E. Azéma, F. Radjaï, A. Taboada, Phys. Rev. E 84, 011306 (2011).

72. B. Saint-Cyr, J.-Y. Delenne, C. Voivret, F. Radjai, P. Sornay, Phys. Rev. E 84, 041302 (2011).

73. Juan Carlos Quezada, Pierre Breul, Gilles Saussine, Farhang Radjai, Phys. Rev. E 86, 031308 (2012).

74. C. Voivret, F. Radjaï, J.-Y. Delenne, M.S. El Youssoufi, Phys. Rev. Lett. 102, 178001 (2009).

75. Dirk Kadau, Guido Bartels, Lothar Brendel, Dietrich E. Wolf, Comput. Phys. Commun. 147, 190 (2002).

76. Ivar Bratberg, Farhang Radjai, Alex Hansen, Phys. Rev. E 66, 031303 (2002)

77. Duc-Hanh Nguyen, Emilien Azéma, Farhang Radjai, Philippe Sornay, Phys. Rev. E 90, 012202 (2014).

78. Duc-Hanh Nguyen, Florian Fichot, Vincent Topin, Nucl. Eng. Des. 313, 96 (2017).

79. Eric Clement, Curr. Opin. Colloid Interface Sci. 4, 294 (1999).

80. GDR-MiDi, Eur. Phys. J. E 14, 341 (2004).

81. J.J. Moreau, Numerical investigation of shear zones in granular materials, in Friction, Arching, Contact Dynam$i c s$, edited by D.E. Wolf, P. Grassberger (World Scientific, Singapore, 1997) pp. 233-247.

82. L. Staron, F. Radjaï, Phys. Rev. E 72, 041308 (2005).

83. Da-Mang Lee, The angles of friction of granular fills, $\mathrm{PhD}$ Thesis, University of Cambridge, 1992.

84. J.P. Bardet, J. Proubet, Géotechnique 41, 599 (1991).

85. J.P. Bardet, J. Proubet, J. Eng. Mech. 118, 397 (1992).

86. A.N.B. Poliakov, H.J. Herrmann, Geophys. Res. Lett. 21, 2143 (1994)

87. H.J. Herrmann, J.A. Astrom, R. Mahmoodi Baram, Physica A: Stat. Mech. Appl. 344, 516 (2004).

88. J. Desrues, G.S. Viggiani, Int. J. Numer. Anal. Methods Geomech. 28, 279 (2004).

89. D.L. Turcotte, J. Geophys. Res.: Solid Earth 91, 1921 (1986).

90. H.J. Herrmann, A.N.B. Poliakov, S. Roux, Fractals 3, 821 (1995).

91. G.R. McDowell, M.D. Bolton, D. Robertson, J. Mech. Phys. Solids 44, 2079 (1996).

92. Tetsuo Akiyama, Keiko M. Aoki, Tatsusaburo Iguchi, Kazuo Nishimoto, Chem. Eng. Sci. 51, 3551 (1996).

93. Leo Rothenburg, R.J. Bathurst, Géotechnique 39, 601 (1989).

94. F. Radjaï, Multicontact dynamics, in Physics of Dry Granular Media, edited by H.J. Herrmann et al. (Kluwer Academic Publishers, Netherlands, 1998) pp. 305-312.

95. H. Ouadfel, L. Rothenburg, Mech. Mater. 33, 201 (2001).

96. E. Azéma, F. Radjaï, R. Peyroux, G. Saussine, Phys. Rev. E 76, 011301 (2007).

97. E. Azema, F. Radjai, B. Saint-Cyr, J.-Y. Delenne, P. Sornay, Phys. Rev. E 87, 052205 (2013).

98. Duc-Hanh Nguyen, Emilien Azéma, Philippe Sornay, Farhang Radjai, Phys. Rev. E 91, 032203 (2015).

99. Farhang Radjai, Vincent Richefeu, Philos. Trans. R. Soc. A: Math. Phys. Eng. Sci. 367, 5123 (2009) 